\title{
RWG Basis Functions for Accurate Modelling of Substrate Integrated Waveguide Slot-based Antennas
}

\author{
Matthieu Bertrand ${ }^{1}$, Guido Valerio ${ }^{1}$, Mauro Ettorre ${ }^{2}$, and Massimiliano Casaletti ${ }^{1}$ \\ ${ }^{1}$ Laboratoire d'Electronique et Électromagnétisme, Sorbonne Université, F-75005 Paris, France \\ ${ }^{2}$ IETR, UMR CNRS 6164, Université de Rennes 1, France
}

\begin{abstract}
In this paper, we propose the implementation of a triangular mesh along with Rao-Wilton-Glisson (RWG) basis functions to improve the performance and flexibility of a mixed Mode-Matching (MM) / Method of Moment (MoM) algorithm dedicated to Substrate Integrated Waveguide (SIW) antennas analysis and optimization. These basis functions represent equivalent magnetic currents on either coupling or radiating apertures. In the initial implementation, entire domain sine basis function were presented, providing fast and accurate simulations for electrically thin rectangular slots. In order to treat wider slots and other geometries, we introduce here RWG functions, present the theoretical framework of this approach and highlight its potential through concrete examples.
\end{abstract}

Index Terms - Method of moments, Mode matching methods, Slot antennas

\section{INTRODUCTION}

$\mathrm{I}_{\mathrm{p} m \mathrm{~s}}^{\mathrm{N}}$ $\mathrm{N}$ recent years, planar microwave and millimeter-wave 1 passive circuits have become increasingly critical parts of communication and imaging systems. In particular, the requirement for low-cost highly directive antennas has drawn attention to the substrate integrated waveguide (SIW)[1] technology. This led to the design of multilayer radiating SIW structures [2] of large electrical dimensions with many small apertures and metallic pins. As a result, the optimization of such structures with commercial full-wave codes is often proving to be a very time consuming and tedious task. While the latest general purpose methods such as in [3] are probably very attractive in this context, we proposed in [4] a dedicated hybrid $\mathrm{MM} / \mathrm{MoM}$ which takes full advantage of the redundant geometrical properties of this structures, such as the presence of only cylindrical diffracting elements. Furthermore, the use of sine-shaped basis functions for the aperture currents provided a very accurate result for electrically thin slot geometries. However, it but was unable to deal with non-rectangular slots, or wide and longer slots exhibiting more complex currents distributions.

In this paper, we propose to add linear basis functions defined on triangular domains [5] (RWG), to deal with such cases. In Section II a general description of the hybrid code is given, followed by a more specific focus on the implementation of the coupling for RWG functions. In Section III, two examples are presented to highlight the advantages and limitations of both basis function types, while comparing the results to that of commercial software. Finally, conclusions are drawn.

\section{THEORETICAL FORMULATION}

\section{A. General considerations}

First, as explained in [3], the code relies on the combination of two numerical methods. The generic single-layer configuration is illustrated in Fig. 1. A MM is used to enforce the boundary conditions on metallic and/or dielectric cylinders

Manuscript received August 1, 2019, revised on October $5^{\text {th }}, 2019$. Corresponding author: M. Bertrand (e-mail: matthieu.bertrand@sorbonneuniversite.fr).

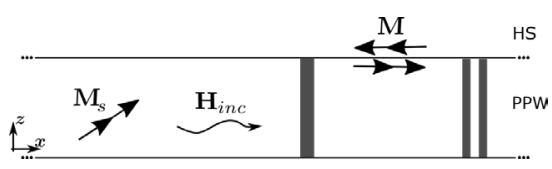

Fig. 1. General configuration for the single layer version of the electromagnetic problem.

connecting horizontal metallic plates. In the meantime, apertures on these metallic plates are represented by equivalent magnetic currents and the fields are solved through the MoM. In this latter procedure, the equivalent currents $\mathbf{M}$ are expressed on a set of $\boldsymbol{N}_{\boldsymbol{b}}$ basis functions $\mathbf{b}_{\mathrm{n}}(\mathbf{r})$ such as:

$$
\mathbf{M}(\mathbf{r})=\sum_{n=1}^{N_{b}} v_{n} \mathbf{b}_{n}(\mathbf{r})
$$

Then, a Galerkin scheme provides the current amplitudes $v_{n}$ by solving the following linear system, where $\underline{\mathbf{Y}}$ is the coupling matrix and $\mathbf{I}$ is the so-called forcing term.

$$
\underline{\mathbf{Y}} \cdot \mathbf{V}=\mathbf{I}
$$

The coupling matrix elements are decomposed into a sum of three terms: the first term is related to the half-space (HS) propagation, the second to the guided parallel-plate waveguide (PPW) modes, and the third results from the diffraction by the posts inside the structure.

$$
\begin{aligned}
& Y_{k, f}=-j \omega \varepsilon_{0}\left(\int_{S} \mathbf{b}_{k}(\mathbf{r}) \cdot \int_{S^{\prime}} \underline{\mathbf{G}}^{H S}\left(\mathbf{r}, \mathbf{r}^{\prime}\right) \cdot \mathbf{b}_{f}\left(\mathbf{r}^{\prime}\right) d S^{\prime} d S\right) \\
& -j \omega \varepsilon\left(\int_{S} \mathbf{b}_{k}(\mathbf{r}) \cdot \int_{S^{\prime}} \underline{\mathbf{G}}^{P P W}\left(\mathbf{r}, \mathbf{r}^{\prime}\right) \cdot \mathbf{b}_{f}\left(\mathbf{r}^{\prime}\right) d S^{\prime} d S\right) \\
& -j \omega \varepsilon\left(\int_{S} \mathbf{b}_{k}(\mathbf{r}) \cdot \int_{S^{\prime}} \underline{\mathbf{G}}^{\text {Posts }}\left(\mathbf{r}, \mathbf{r}^{\prime}\right) \cdot \mathbf{b}_{f}\left(\mathbf{r}^{\prime}\right) d S^{\prime} d S\right)
\end{aligned}
$$

For each integral term, the proper Green's function for magnetic currents is employed, i.e. $\underline{\mathbf{G}}^{H S}, \underline{\mathbf{G}}^{P P W}$, or $\underline{\mathbf{G}}^{\text {Posts }}$, respectively. Test and source currents domain are $S^{\prime}$ and $S$, while $\varepsilon_{0}$ and $\varepsilon$ are the vacuum and substrate electrical permittivities, and $\omega$ is the angular frequency. 


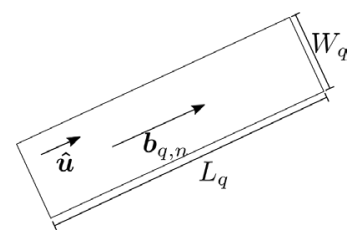

(a)

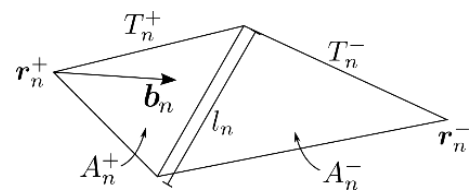

(b)
Fig. 2. (a) ED basis function defined on an entire slot, (b) RWG basis function defined on a pair of mesh triangles defined inside a slot.

Finally, the forcing terms are the dot products of the incident magnetic field $\mathbf{H}_{i n c}$ and test functions. $\mathbf{H}_{i n c}$ is produced by a coaxial or waveguide source represented by a equivalent magnetic current $\mathbf{M}_{\mathrm{s}}$ in the absence of slots, it is computed through the SIW Green's function, which is the sum of $\underline{\mathbf{G}}^{P P W}$ and $\underline{\mathbf{G}}^{\text {Posts }}([3])$.

$$
\begin{aligned}
& i_{k}=\int_{S} \mathbf{b}_{k}(\mathbf{r}) \cdot \mathbf{H}_{\text {inc }}(\mathbf{r}) d S \\
& =\int_{S} \mathbf{b}_{k}(\mathbf{r}) \cdot\left(-j \omega \varepsilon \int_{\text {Source }} \underline{\mathbf{G}}^{S I W}\left(\mathbf{r}, \mathbf{r}^{\prime}\right) \cdot \mathbf{M}_{S}\left(\mathbf{r}^{\prime}\right) d \mathbf{r}^{\prime}\right) d S
\end{aligned}
$$

The initial entire domain (ED) basis function set was based on a sine expansion, in particular, the current on the $q$-th slot was written as:

$$
\mathbf{b}_{q, n}(\mathbf{r})=\hat{\mathbf{u}} \frac{1}{W_{q}} \sin \left[\frac{n \pi}{L_{q}}\left(u+\frac{L_{q}}{2}\right)\right] .
$$

Note that the current (5) is directed along the slot length $L_{q}(u$ axis) without variation along its width $W_{q}$, as shown on Fig. 2(a).

In this paper, we propose to hybridize the basis function set by adding piecewise linear variations on triangular domains, commonly known as RWG functions [5]. In particular, a basis function $\mathbf{b}_{\mathbf{n}}$ is defined on two adjacent triangles $T_{n}{ }^{+}$and $T_{n}{ }^{-}$, of respective areas $A_{n}{ }^{+}$and $A_{n}{ }^{-}$, sharing a common edge of length $l_{n}$. In each triangle, the vertex diametrically opposed to the common edge is denoted $\mathbf{r n}_{\mathbf{n}}^{ \pm}$. Suitable normalization constants ensure the continuity of the current component normal to the common edge:

$$
\mathbf{b}_{n}(\mathbf{r})= \begin{cases}\frac{l_{n}}{2 A_{n}^{+}}\left(\mathbf{r}-\mathbf{r}_{n}^{+}\right) & \text {if } \mathbf{r} \in T_{n}^{+} \\ -\frac{l_{n}}{2 A_{n}^{-}}\left(\mathbf{r}-\mathbf{r}_{n}^{-}\right) & \text {if } \mathbf{r} \in T_{n}^{-}\end{cases}
$$

A geometrical description of (6) is given in Fig. 2 (b).

\section{B. Coupling matrix computation}

We will focus here on the RWG basis functions, which constitute the novel part of this work. RWG basis functions are defined on pairs of triangles, so that each triangle can be part of up to three basis functions, inducing redundancy in the coupling terms. To avoid useless computation, the coupling is therefore computed between triangles. We will mainly deal here with the HS and PPW contributions, as the post diffraction is handled in the exact same way as in [4].

Two terms, $C_{\alpha, \beta}^{s, H S / P P W}$ and $C_{\alpha, \beta, i, j}^{v, H S / P P W}$, related to both scalar and vector potentials are defined as follows:

$$
\begin{gathered}
C_{\alpha, \beta}^{s, H S / P P W}=\int_{T_{\alpha}} \int_{T_{\beta}} g^{H S / P P W}\left(\mathbf{r}, \mathbf{r}^{\prime}\right) d S d S^{\prime} \\
C_{\alpha, \beta, i, j}^{v, H S / P P W}=\int_{T_{\alpha} T_{T_{\beta}}}\left[\Lambda_{\alpha}^{i}(\mathbf{r}) \cdot \Lambda_{\beta}^{j}\left(\mathbf{r}^{\prime}\right)\right] g^{H S / P P W}\left(\mathbf{r}, \mathbf{r}^{\prime}\right) d S d S^{\prime}
\end{gathered}
$$

In these equations, $\alpha$ and $\beta$ denote the triangles $T_{\alpha}$ and $T_{\beta}$ while $i, j \in[1,3]$ indicates the considered free vertices. Finally, denormalized linear basis functions are defined as $\Lambda_{\alpha}^{i}(\mathbf{r})=\mathbf{r}-\mathbf{r}_{\alpha}^{i}$ and $\Lambda_{\beta}^{j}(\mathbf{r})=\mathbf{r}-\mathbf{r}_{\beta}^{j}$, while $g^{H S / P P W}\left(\mathbf{r}, \mathbf{r}^{\prime}\right)$ is the scalar Green's function. To obtain (7)-(8) these equations, the differential operator $\nabla$ was transferred from the dyadic Green's function onto the basis and test functions through integrations by parts. The constants resulting from the differentiation of RWG linear basis functions are added later in the recombination of the two potential contributions:

$$
C_{\alpha, \beta, i, j}^{H S / P P W}=l_{\alpha}^{i} l_{\beta}^{j}\left(-j \omega \varepsilon \frac{C_{\alpha, \beta, i, j}^{v, H S / P P W}}{4 A_{\alpha} A_{\beta}}+\frac{j}{\omega \mu} \frac{C_{\alpha, \beta}^{s, H S / P P W}}{A_{\alpha} A_{\beta}}\right)
$$

In this equation, $l_{\alpha}^{i}$ and $l_{\beta}^{j}$ stand for the length of the edges directly opposite to the free vertices $\mathbf{r}_{\alpha}^{i}$ and $\mathbf{r}_{\beta}^{j}, A_{\alpha}$ and $A_{\beta}$ are the areas of $T_{\alpha}$ and $T_{\beta}$, while $\varepsilon$ and $\mu$ refer to the considered medium permittivity and permeability, respectively. Finally, the MoM matrix is obtained by adding the proper contributions for each basis function pair.

We will now focus on the different methods implemented to compute the integrals (7)-(8). Depending on the distance between the coupled elements, spatial, spectral or asymptotic representations were adopted. First, let us consider the coupling resulting from half-space radiation. The singular integrals appearing in self or adjacent couplings are treated as in [6]. In particular, the divergence theorem is applied twice in order to transform the two surface integrals on the triangles to a numerical integration on their edges of an analytical integrand. These transformations also cancel the singularity in $1 / R$. For non-singular couplings, a straightforward triangular Gauss-Legendre integration is applied with the Green's function spatial representation given by

$$
g^{H S}\left(\mathbf{r}, \mathbf{r}^{\prime}\right)=\frac{e^{-j k\left|\mathbf{r}-\mathbf{r}^{\prime}\right|}}{2 \pi\left|\mathbf{r}-\mathbf{r}^{\prime}\right|}
$$

(a factor 2 is added to take into account the presence of the infinite plane on which the source is lying.)

Secondly, we will give more insight into the coupling related to the field excited in the PPW regions. By applying the equivalence theorem iteratively, a magnetic current source between two metallic planes can be replaced by an infinite periodic array of sources having doubled amplitude separated by twice the waveguide thickness $h$. All of these sources radiate in free space so that the total scalar Green's function is given by:

$$
g^{P P W}\left(\mathbf{r}, \mathbf{r}^{\prime}\right)=\sum_{n=-\infty}^{+\infty} g^{H S}\left(\mathbf{r}, \mathbf{r}^{\prime}+2 n h \hat{\mathbf{z}}\right)
$$

One should pay attention to the $n=0$ term, which is singular for all triangles sharing one or more vertex, and it is here treated again as in [6]. This formulation is particularly suited for 
close coupling, as the lowest order terms dominate the sum. For couplings at greater distances, the following spectral expansion involving a few cylindrical waveguide modes is more suitable:

$$
\begin{aligned}
& g^{P P W}\left(\mathbf{r}, \mathbf{r}^{\prime}\right)=\frac{1}{8 j h} \sum_{m=-\infty}^{\infty} H_{0}^{(2)}\left(k_{\rho m}\left|\mathbf{r}-\mathbf{r}^{\prime}\right|\right) \\
& k_{\rho m}=\left\{\begin{array}{l}
\sqrt{k^{2}-k_{z, m}^{2}}, k^{2}>k_{z, m}^{2} \\
-j \sqrt{k_{z, m}^{2}-k^{2}}, k^{2}<k_{z, m}^{2}
\end{array}, k_{z, m}=\frac{m \pi}{h}\right.
\end{aligned}
$$

This last formulation is particularly efficient for thin waveguides, in which the higher-order modes are rapidly attenuated. Again, for representations (11) and (12) a Gauss-Legendre integration is adopted. Finally, an asymptotic approximation of the integrals was implemented for the couplings at large distances. The coupling is first expressed in the spectral domain, the vector between the coupled triangles centroids is $\boldsymbol{\rho}_{\alpha \beta}=\rho_{\alpha \beta} \hat{\boldsymbol{\rho}}_{\alpha \beta}, \eta$ is the wave impedance, and the wavenumber is written as $\mathbf{h}=h_{x} \hat{\boldsymbol{\rho}}_{\alpha \beta}+h_{y}\left(\hat{\mathbf{z}} \times \hat{\boldsymbol{\rho}}_{\alpha \beta}\right)$ :

$$
C_{\alpha, \beta, i, j}^{P P W}=\frac{1}{(2 \pi)^{2}} \int_{-\infty}^{+\infty} \int_{-\infty}^{+\infty} M(\mathbf{h}) e^{-j \mathbf{h} \cdot \hat{\boldsymbol{\rho}}_{\alpha \beta} \rho_{\alpha \beta}} \tilde{g}^{P P W}(\mathbf{h}) d \mathbf{h}
$$

where $M(\mathbf{h})$ is given by

$$
M(\mathbf{h})=\frac{l_{\alpha}^{i} l_{\beta}^{j}}{4 A_{\alpha} A_{\beta}} \tilde{\Lambda}_{\alpha, i}(-\mathbf{h}) \cdot\left(\underline{\mathbf{1}}^{2}-\mathbf{h} \mathbf{h}\right) \cdot \tilde{\Lambda}_{\beta, j}(\mathbf{h})
$$

and the Green's function Fourier transform by

$$
\tilde{g}^{P P W}(\mathbf{h})=\frac{1}{j h k \eta} \sum_{m=0}^{\infty} \frac{\varepsilon_{m}}{k_{\rho m}^{2}-\mathbf{h} \cdot \mathbf{h}}
$$

where $\varepsilon_{m}$ is 1 for $m=0$ and 2 otherwise.

Then, adapting the derivations from [7] to the RWG case and neglecting attenuated higher-order modes, the coupling reduces to the integral (16), evaluated by a Gauss-Hermite integration.

$$
\begin{aligned}
& C_{\alpha, \beta, i, j}^{P P W}=-\frac{e^{-j k_{\rho 0} \rho_{\alpha \beta}}}{\sqrt{2 j} 2 \pi j h k \eta} \times \\
& \int_{-\infty}^{+\infty}\left(\frac{M\left(k_{\rho 0}\left(1-j s^{2}\right), k_{\rho 0} s \sqrt{2 j+s^{2}}\right)}{\sqrt{1-j s^{2} / 2}}\right) e^{-k_{\rho 0} \rho_{\alpha \beta} s^{2}} d s
\end{aligned}
$$

\section{NUMERICAL RESULTS AND VALIDATION}

In order to evaluate its accuracy, the code was used to compute the field radiated by two previously realized large antennas, a radial line slot antenna (RLSA) [8], and a dual reflector leaky-wave antenna (LWA) [9], depicted in Fig. 2. The first antenna is composed of radially arranged radiating slots fed by a single coaxial feed in its center. It was designed in [8] to radiate a specific distribution of $z$-oriented electrical field in the near field. The second antenna radiates a pencil beam in the far field. Two coaxial sources on the left side of the structure in Fig. 2(b) generate an impinging wave on a Gregorian system feeding the arrays of slots. Both structures exhibit a large number of either metallic posts, slots, or both, making them interesting test structures for the developed numerical tool.

A commercial FEM code (HFSS) and a time domain solver based on Finite Integration Technique (CST) were also used for comparison. However, we were unable to obtain a proper

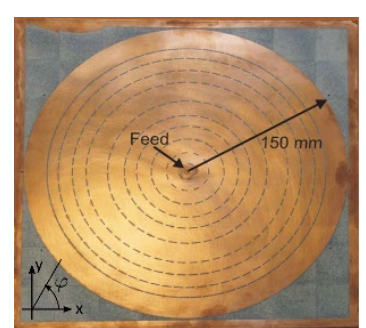

(a)

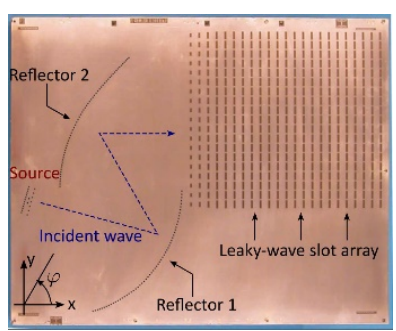

(b)
Fig 3. Photograph of the simulated antennas, (a) RLSA, (b) LWA, respectively from [8] and[9].

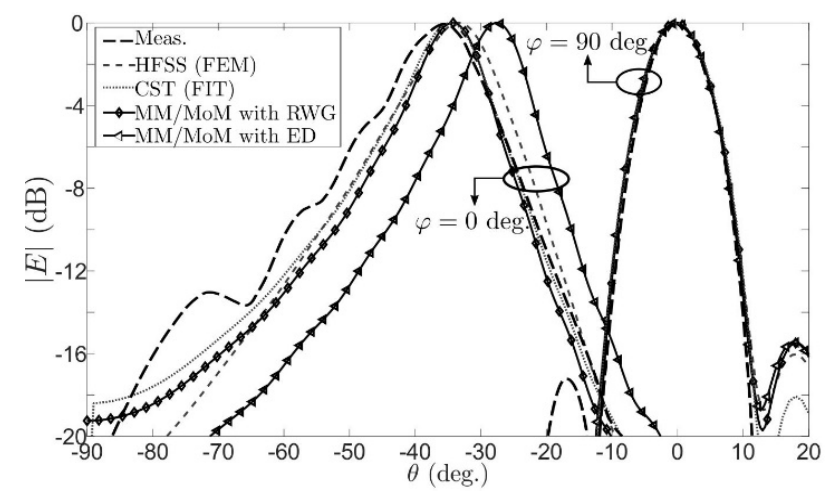

Fig 5. Normalized LWA far field, with measurement data extracted from [9]

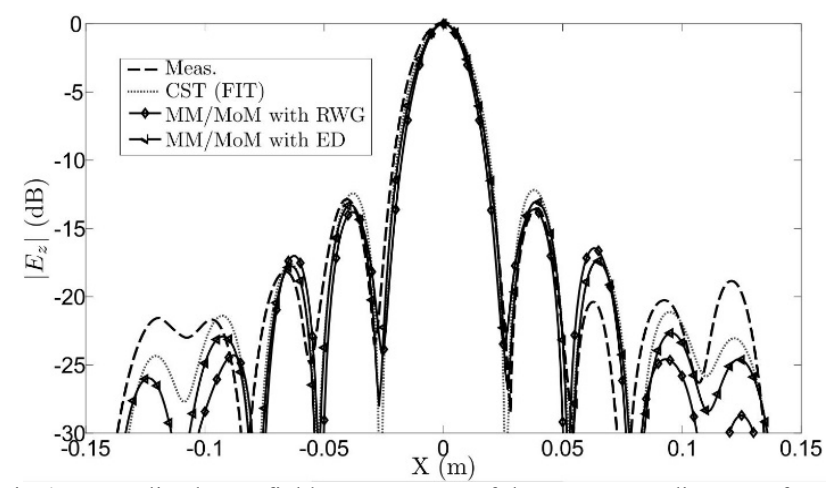

Fig 4. Normalized near-field $z$-component of the RLSA at a distance of $150 \mathrm{~mm}$, along with measurement data extracted from [8].

evaluation of the near field radiated by the RLSA with HFSS FEM method due to the required large airbox needed to evaluate the field, introducing resonances and a poor field evaluation. For all simulations, we used an Intel-Core i7-3770 CPU at $3.40 \mathrm{GHz}$ with $32 \mathrm{~GB}$ of RAM.

\section{A. Accuracy}

Starting with the RLSA antenna, the normalized $z$ component evaluated at a distance of $150 \mathrm{~mm}$ of the antenna plane is illustrated in Fig. 3. All methods provide a good estimation of the pattern, especially for the three main lobes. The differences on the lateral lobes can be attributed to fabrication tolerances and edge diffraction effects. While the measured antenna is offinite size, our code assumes an infinite structure, while CST approximates an absorbing boundary to imitate free space propagation.

Secondly, the different evaluations of the radiated normalized far field of the LWA, as well as the measurement results, are presented in Fig. 4. Due to the strong asymmetry of 
TABLE I

TIME AND MEMORY CONSUMPTION

\begin{tabular}{|c|c|c|c|c|c|}
\hline & & \multicolumn{2}{|c|}{ Commercial tools } & \multirow{2}{*}{$\begin{array}{c}\text { This work } \\
\text { (RWG) }\end{array}$} & \multirow{2}{*}{$\begin{array}{c}\text { This work } \\
\text { (ED) }\end{array}$} \\
\hline & & HFSS & CST & & \\
\hline \multirow{2}{*}{ RLSA } & Time & -- & $9 \mathrm{~h} 21 \mathrm{~min}$ & $28 \mathrm{~min}$ & $5 \min 20 \mathrm{~s}$ \\
\hline & RAM & -- & $10.7 \mathrm{~GB}$ & $20.1 \mathrm{~GB}$ & $2.2 \mathrm{~GB}$ \\
\hline \multirow{2}{*}{ LWA } & Time & $45 \mathrm{~min}$ & $37 \mathrm{~h}$ & $4 \min 45 s$ & $3 \min 25 \mathrm{~s}$ \\
\hline & RAM & $21.3 \mathrm{~GB}$ & $6.2 \mathrm{~GB}$ & $10 \mathrm{~GB}$ & $3.5 \mathrm{~GB}$ \\
\hline
\end{tabular}

the pattern, it is here calculated in the two cut planes defined in spherical coordinates by $\varphi=0$ degrees and $\varphi=90$ degrees.

In this particular case of relatively large slots, the ED basis functions do not produce the correct maximum directivity angle. On the contrary, one can see that the response with RWG functions is much closer to the measured data. It is also in agreement with the results provided by commercial software, in particular with the time domain solver CST.

When comparing the accuracy of the solutions, one should keep in mind that the proper evaluation of the post diffraction in both HFSS and CST depend on the accuracy of the 3D mesh, the former being hexahedral, the latter cubic. While the chosen mesh is important also with RWG basis functions to describe currents on slots - in this case, 18 of them are sufficient to provide these results -, the post contribution is exact, as the full cylindrical shape is included analytically in the MM boundaries.

\section{B. Timing and memory comparisons}

The computation time of all simulations, as well as the memory consumptions, is summarized in Table I. Considering first of all the time required to solve each antenna problem, it is clear that the MM/MoM is much faster than the commercial codes in both cases. Starting with the RLSA, the ED provides in less than 6 minutes the pattern given by CST after more than 9 hours of simulation. In this case, the RWG offer also a significant time gain but it requires more basis functions, indicating that resonant slots are indeed more easily represented on the sine basis. For the LWA, the RWG set provides the solution in less than 5 minutes, compared to 45 minutes in HFSS and 37 hours in CST. The ED computation is even faster but is unable to reach the physical solution.

As far as memory is concerned, the ED formulation is always more advantageous because of its simplicity. In the RLSA case, ED only requires $2.2 \mathrm{~GB}$ of RAM for the RLSA, while the RWG formulation needs $20.1 \mathrm{~GB}$ and CST $10.7 \mathrm{~GB}$. For the LWA case, HFSS (which is able to solve the problem in a reasonable time) requires $21.3 \mathrm{~GB}$ of memory, versus $10 \mathrm{~GB}$ and $3.5 \mathrm{~GB}$ needed by the RWG and ED basis functions, respectively. Overall, the $\mathrm{MM} / \mathrm{MoM}$ still requires a decent amount of memory for large antennas but using the proper basis functions one can expect to about half the memory burden of current commercial codes.

\section{C.Discussion}

Beyond a straightforward comparison of the memory usage and time consumption, several points will be discussed here that are essentials for the optimization of large SIW antennas.

First, while a simulation with a single set of geometrical parameters indicates how fast the method can be, it hides the fact that during an optimization procedure, only a few of them would be modified at each iteration. This is significant because in this solution only the relevant couplings would then be computed again. On the contrary, a 3D FEM approach as in HFSS or CST would require a complete re-meshing.

Second, while the commercial software can only deal with one or two symmetry planes, this hybrid code takes into account further symmetries, by recycling all identical couplings. This significantly reduces the computation time for antennas with some type of periodicity, as in the cases presented here.

Third, in many beam-forming networks SIW solutions, pins are used in large number to create reflectors following the quasi-optics principles. This leads to geometries such as the one presented in this paper, where the coupling between slots induced from the diffraction of the pins is most of the time negligible compared to the half-space and parallel-plate waveguide contributions. Knowing this, a significant time can be saved in the computation.

\section{CONCLUSION}

We presented the hybridization of the basis functions in a $\mathrm{MM} / \mathrm{MoM}$ code for SIW antennas. With the addition of RWG functions providing a better description of the currents, the numerical tool is now able to deal with electrically large slots, but also any kind of non-rectangular geometries. A significant time and resource reduction can be obtained in comparison with commercial solutions. The authors intend to demonstrate in future works the code potential for large antenna optimizations.

\section{V.ACKNOWLEDGMENT}

The authors would like to thank the Direction Générale de l'Armement (DGA) for its financial support through the ANR ASTRID Fast-HEM-3D project. We would also like to thank the authors of [8] and [9] used as a basis for the numerical comparison done in this paper.

\section{REFERENCES}

[1] D. Deslandes and K. Wu, "Integrated microstrip and rectangular waveguide in planar form," IEEE Microw. Wireless Compon. Lett., vol.11, no. 2, pp. 68-70, Feb. 2001.

[2] J. Xu, Z. N. Chen, X. Qing and W. Hong, "140-GHz TE20-mode dielectric-loaded SIW slot antenna array in LTCC," IEEE Trans. Antennas Propag., vol. 61, no. 4, pp. 1784-1793, April 2013.

[3] R. Torchio, P. Alotto, P. Bettini, D. Voltolina and F. Moro, "A 3-D PEEC formulation based on the cell method for full-wave analyses with conductive, dielectric, and magnetic media," in IEEE Trans. Magnetics, vol. 54, no. 3, pp. 1-4, March 2018, Art no. 7201204.

[4] M. Casaletti, G. Valerio, J. Seljan, M. Ettorre, and R. Sauleau, "A fullwave hybrid method for the analysis of multilayered SIW based antennas," IEEE Trans. Antennas Propag., vol. 61, no. 11, pp. 5575-5588, Nov. 2013.

[5] S. Rao, D. Wilton, and A. Glisson, "Electromagnetic scattering by surfaces of arbitrary shape," IEEE Trans. Antennas Propag., vol. 30, no. 3, pp. 409-418, May 1982.

[6] D. R. Wilton, F. Vipiana, and W. A. Johnson, "Evaluation of 4-D reaction integrals in the method of moments: coplanar element case ", IEEE Trans. Antennas Propag., vol. 65, no 5, p. 2479 2493, mai 2017.

[7] M. Albani, A. Mazzinghi, and A. Freni, "Asymptotic approximation of mutual admittance involved in MoM analysis of RLSA antennas," IEEE Trans. Antennas Propag., vol. 57, no. 4, pp. 1057-1063, April 2009.

[8] M. Ettorre et al., "On the near-field shaping and focusing capability of a radial line slot array," IEEE Trans. Antennas Propag., vol. 62, no. 4, pp. 1991-1999, April 2014.

[9] M. Ettorre, A. Neto, G. Gerini and S. Maci, "Leaky-wave slot array antenna fed by a dual reflector system," IEEE Trans. Antennas Propag., vol. 56, no. 10, pp. 3143-3149, Oct. 2008.. 